\section{Entstehungsmechanismus schwarzer Metallaufdampfschichten}

\author{
Von Yoshiniko Mizushima \\ Electrical Communication Laboratory \\ Musashino-shi, Tokio, Japan
}

(Z. Naturforschg. 16 a, 1260-1261 [1961] ; eingegangen am 5. Juni 1961)

Der Entstehungsmechanismus schwarzer Metallschichten, die unter Restgasdruck aufgedampft werden, ist noch nicht geklärt. Kürzlich haben Fritsche et al. ${ }^{1}$ über Wismutruß einige interessante Deutungen mitgeteilt. $\mathrm{Da}$ verschiedenartige Metalle ( $\mathrm{Au}, \mathrm{Ag}, \mathrm{Bi}, \mathrm{Pb}, \mathrm{Cd}, \mathrm{Zn}$, $\mathrm{Sb}, \mathrm{Sn}$ ) unter verschiedenen Restgasen (Luft, $\mathrm{N}_{2}, A$ ) verschwärzt werden können, müßte man einen sehr allgemeinen Mechanismus annehmen.

Beim Aufdampfen von $\mathrm{Au}$ in A wurde eine wolkenförmige Strömung beobachtet ${ }^{2}$, was mit der Angabe von Fritsche in vollkommenem Einklang steht. Die schwarze Schicht zeigte beim Elektronenbeugungsversuch keinerlei Orientierung und haftete elektronenmikroskopisch nicht fest am Substrat. Das beweist, daß die Goldkristalle schon in der Gasphase gebildet und nacheinander auf das Substrat gehäuft werden. Die Kristallitgröße war mit über $100 \AA$ nicht kleiner als beim Vakuumaufdampfen.

Der „kritische“ Gasdruck für die Entstehung der schwarzen Schicht liegt zwischen 0,1 und 1 Torr (Bi/Luft 0,2 Torr, $\mathrm{Au} / \mathrm{A}$ 0,5 Torr, $\mathrm{Ba} / \mathrm{A}$ 0,8 Torr, $\mathrm{Au} / \mathrm{N}_{2}$ 0,3 Torr $\left.^{3}\right)$. Die freie Weglänge der Gasmolekeln ist ca. $10^{-3} \mathrm{~cm}$, was zeigt, daß einmalige Stöße für den Mechanismus nicht ausreichen.

Die Stoßzahl $\left(Z_{2}\right)$ zwischen zwei Gasatomen mit den Molekulargewichten $M_{1}, M_{2}$, den Atomdurchmessern $d_{1}, d_{2}$ und den Teilchendichten $N_{1}, N_{2}$ ist

$$
Z_{2}=2 \sqrt{2 \pi} N_{1} N_{2}\left(\frac{d_{1}+d_{2}}{2}\right)^{2} \sqrt{\frac{M_{1}+M_{2}}{M_{1} M_{2}}} R T .
$$

Für eine einzige Atomart gilt

$$
2 Z_{2}=4 \sqrt{\pi} N^{2} d^{2} \cdot \sqrt{R T / M} .
$$

Wenn der Stoßkomplex eine Lebensdauer $\tau_{2}$ hat, dann wird die Tripelstoßzahl

$$
Z_{3}=2 \sqrt{2 \pi} N Z_{2} \tau_{2}\left(\frac{d+d_{2}}{2}\right)^{2} \sqrt{\frac{M+M_{2}}{M M_{2}}} R T .
$$

Im folgenden bezeichnet das Suffix einen $n$-fachen Komplex. Es gilt

$$
\begin{aligned}
\frac{Z_{n+1}}{Z_{n}}=2 \sqrt{2 \pi} N \tau_{n}\left(\frac{d+d_{n}}{2}\right)^{2} \sqrt{\frac{M+M_{n}}{M M_{n}}} R T \\
=\left(\frac{1}{\sqrt{2}} \sim 4\right) \sqrt{\pi} N \tau_{n} d_{n}^{2} \sqrt{R T / M},
\end{aligned}
$$

${ }^{1}$ L. Fritsche, F. Wolf u. A. Schaber, Z. Naturforschg. 16a, 31 [1961].

2 Y. Mizushima u. Z. Oda, J. Phys. Soc., Japan 12, 355 [1957]. - S. Hirota, Y. Mizushima u. H. Noake, J. Appl. Phys., Japan 25, 294 [1956].

3 L. Harris, D. Jeffries u. B. M. Siegel, J. Appl. Phys. 19, 791 [1948]. wobei die Zahlen in der letzten Klammer zu den Extremfällen, $M \ll M_{n}, d \ll d_{n}$ und $M \approx M_{n}, d \approx d_{n}$, gehören. Die freie Weglänge und die mittlere Geschwindigkeit sind in elementarer Form

$$
\lambda=1 / \sqrt{2} \pi N d^{2} \text { und } u=(\sqrt{3} \sim \sqrt{2}) \sqrt{R T / M} .
$$

Man erhält also

$$
Z_{n+1} / Z_{n} \approx \tau_{n} u / \lambda
$$

Wendet man sie auf den Metallkomplex an, so liefert sie als notwendige Bedingung für das Wachsen der Stoßkomplexe

$$
Z_{n+1} / Z_{n} \geq 1, \quad \text { d. h. } \quad \tau_{n} \geq \lambda / u .
$$

Bei den oben erwähnten Fällen ist $\lambda=10^{-3} \sim 10^{-2}$ $\mathrm{cm}$ und $u=10^{4} \sim 10^{5} \mathrm{~cm} / \mathrm{s}$ bei $T \approx 1000^{\circ} \mathrm{K}$. Somit wird $\tau_{n} \approx 10^{-7} \mathrm{~s}$ und hängt nur schwach von $n$ und vom Metall $\mathrm{ab}$, da bei Änderung von $M$ (und $d$ ) $\lambda$ und $u$ einander parallel variieren.

Da $\tau=\tau_{0} \exp (Q / R T)$ und bei normalen Diatomen die Schwingungsfrequenz $100 \sim 300 \mathrm{~cm}^{-1}$ ist, kann man $\tau_{0}=10^{-13} \sim 10^{-12}$ ansetzen. Als $Q$ nimmt man annähernd die Dissoziationsenergie $D$ des Diatoms an ${ }^{4}$. Bei großem $n$ steigt $Q$ allmählich und nähert sich der Sublimationsenergie $L$ des Metalls.

Damit läßt sich die „kritische“ Temperatur $T_{\mathrm{k}}$ für die Entstehung schwarzer Aufdampfschichten grob ermitteln, wie es in Tab. 1 gezeigt ist.

\begin{tabular}{|l|c|c|c|c|c|}
\hline & $\begin{array}{c}D \\
\mathrm{kcal}\end{array}$ & $\begin{array}{c}T_{\mathrm{k}} \\
{ }^{\circ} \mathrm{K}\end{array}$ & $\begin{array}{c}L \\
\mathrm{kcal}\end{array}$ & $\begin{array}{c}T_{\mathrm{V}} \\
{ }^{\circ} \mathrm{K}\end{array}$ & $\begin{array}{c}T_{\mathrm{a}} \\
{ }^{\circ} \mathrm{K}\end{array}$ \\
\hline $\mathrm{Au}$ & 53 & 1900 & 82 & 1700 & 1900 \\
$\mathrm{Bi}$ & 39 & 1400 & 43 & 1000 & 1200 \\
$\mathrm{~Pb}$ & 14 & 500 & 42 & 1000 & 1200 \\
$\mathrm{Zn}$ & 6 & 250 & 28 & 600 & 800 \\
\hline
\end{tabular}

Tab. l. Abschätzung der kritischen Temperatur für Entstehung schwarzer Metallaufdampfschichten.

$T_{\mathrm{a}}$ zu $200{ }^{\circ} \mathrm{K}$ höher angenommen als $T_{\mathrm{v}}$.

Die ausgerechnete $T_{\mathrm{k}}$ muß nicht höher sein als die Temperatur der Aufdampfquelle $T_{\mathrm{a}}$, die etwas höher wird als die Vakuumaufdampftemperatur $T_{\mathrm{v}}$.

Bei $\mathrm{Au}$ und $\mathrm{Bi}$ ist $T_{\mathrm{k}} \approx T_{a}$, was bedeutet, daß der Stoßkomplex dicht neben der Verdampfungsquelle entsteht. Bei $\mathrm{Pb}$ und $\mathrm{Zn}$ ist $T_{\mathrm{k}}<T_{\mathrm{a}}$, was bedeutet, daß der Komplex in der Nähe der Quelle unstabil ist und etwas entfernt davon entsteht.

Die oben entwickelte Hypothese beschreibt nur eine notwendige Bedingung für die Entstehung der schwarzen Schichten. Eine andere Bedingung ist, daß genü-

4 A.G. Gaydon, Dissociation Energies and Spectra of Diatomic Molecules, Chapman \& Hall Ltd., London 1953. - Y. K. Syrkin u. M. E. Dyatrina, Structure of Molecules and the Chemical Bond, Butterworths Sci. Publ., London 1950. P. Schissed, J. Chem. Phys. 26, 1276 [1957]. 
gend viele Metallatome nachgeliefert werden, so daß der Verlust durch Dissoziation des Komplexes immer wieder reichlich ersetzt wird. Fritsche et al. schreiben der Auftreffrate den Einfluß zu, daß eine große Auf-

\section{Zur Defektelektronenleitung in aufgedampften Germanium-Schichten}

\section{Von Herbert Pagnia}

Physikalisches Institut der Technischen Hochschule Darmstadt (Z. Naturforschg. 16 a, 1261-1262 [1961] ; eingeg. am 4. September 1961)

Electrical conductivity and $\mathrm{H}_{\mathrm{ALL}}$-effect measurements were made with Germanium films, vacuum evaporated on fused quartz plates having temperatures of about $450^{\circ} \mathrm{K}$. Film thicknesses range from 400 to $2000 \AA$. Mean hole concentration was of the order $3,5 \cdot 10^{18} / \mathrm{cm}^{3}$ with a weak dependency on film thickness. Mobility changes stronger. The concentration of the light and heavy holes were determined from the dependency of HaLL-coefficient on magnetic force. The quotient of these concentrations was 0,045 . Qualitatively there was satisfactory agreement with p-type monocrystalline Germanium values.

Das Bändermodell des Germaniums ist heute sehr gut bekannt ${ }^{1}$. Während das Leitungsband eine komplizierte anisotrope Struktur aufweist, können die beiden zur Leitfähigkeit beitragenden Valenzbänder in erster Näherung als isotrop angesehen werden. Daher sollten Effekte, die im wesentlichen nur von den beiden Defektelektronenarten mit verschiedenen effektiven Massen bewirkt werden, bei ein- und polykristallinen Materialien qualitativ die gleichen Abhängigkeiten zeigen.

Für einen solchen Vergleich schienen dünne Germanium-Aufdampfschichten geeignet, da sie bei der Herstellung auf nichtkristalliner Unterlage in einem bestimmten Temperaturbereich des Trägers polykristallin aufwachsen ${ }^{2}$ und ausnahmslos eine große Defektelektronenkonzentration haben ${ }^{3-5}$.

Zur Untersuchung wurden Germanium-Schichten im Vakuum bei etwa $10^{-5}$ Torr auf geheizte Quarzglasträger mit Temperaturen zwischen 410 und $450^{\circ} \mathrm{K}$ aus einer Wolframwendel aufgedampft. Als Aufdampfmaterial diente durch Zonenschmelzen gereinigtes Germanium. Elektronenbeugungsaufnahmen von Vergleichsschichten auf SiO-Häuten zeigten scharfe Germaniumringe; die elektronenmikroskopische Untersuchung ergab mittlere Kristallitabmessungen von etwa $500 \AA$, d. h. von der Größenordnung der Schichtdicke.

1 O. Madelung, Handb. f. Physik 20, 1 [1957].

2 J. E. DaveY, J. Appl. Phys. 52, 877 [1961].

3 J. W. Thornhill u. K. Lark-Horowitz, Phys. Rev. 82, 762 [1951].

${ }^{4}$ G. A. Kurov, S. A. Semiletov u. Z. G. Pinsker, Dokl. Akad. dampfgeschwindigkeit die Verschwärzung begünstigt. Dies ist sicher wichtig bei $\mathrm{Pb}, \mathrm{Zn}$ und wahrscheinlich $\mathrm{Ba}$, wo der Komplex im dynamischen Gleichgewicht entsteht.

Die Schichten hatten einen über zwei Zehnerpotenzen kleineren spezifischen elektrischen Widerstand als das Ausgangsmaterial. Dies deutet bei sicherlich verkleinerter Beweglichkeit auf eine wesentlich höhere Trägerkonzentration hin. Dementsprechend sind die HALL-Konstanten dieser Schichten kleiner als bei massivem einkristallinem Material, jedoch von der gleichen Größe wie bei dünnen einkristallinen Schichten ${ }^{4}$ (etwa $3 \mathrm{~cm}^{3} / \mathrm{As}$ ). Der Einfluß der Schichtdicke auf die im Vakuum gemessene HALL-Konstante ist nur gering (Abb. 1). Ebenso erwies sich der Einfluß der Unterlagentemperatur bei der Herstellung in einem Bereich von 410 bis $450^{\circ} \mathrm{K}$ als unmeßbar klein.

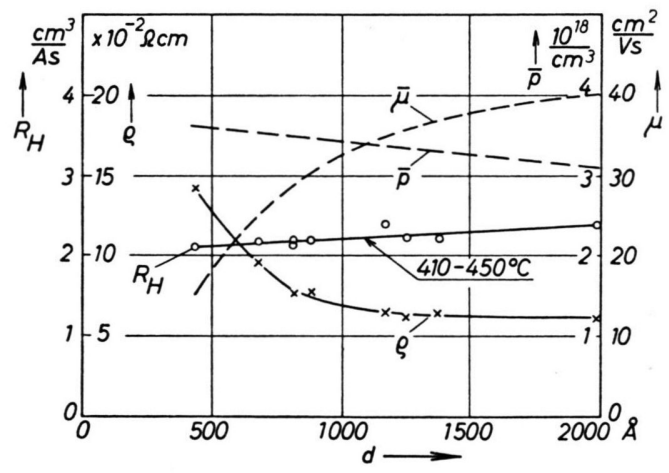

Abb. 1. Abhängigkeit der $\mathrm{H}_{\mathrm{ALL}}-\mathrm{Konstanten} R_{\mathrm{H}}$ und des spezifischen elektrischen Widerstandes $\varrho$ sowie der daraus berechneten mittleren Defektelektronen-Konzentrationen $\bar{p}$ und -Beweglichkeiten $\bar{\mu}$ von der Schichtdicke $d$ (Kondensationstemperatur 410 bis $450^{\circ} \mathrm{C}$ ).

Verzichtet man zur Abschätzung der Trägerkonzentration zunächst auf die Unterscheidung der beiden Defektelektronenarten, so erhält man für die mittlere Defektelektronenkonzentration $\bar{p}$ nach

$$
R_{\mathrm{H}}=\left(U_{\mathrm{H}} d\right) /\left(B_{z} I_{x}\right)=f /(e \bar{p})
$$

$\left(R_{\mathrm{H}}=\right.$ Hall$_{\mathrm{AL}}-$ Konstante, $U_{\mathrm{H}}=\mathrm{H}_{\mathrm{ALL}}-\mathrm{Spannung}, d=$ (interferometrisch ${ }^{6}$ gemessene) Schichtdicke, $B_{z}=$ magnetische Kraftflußdichte in Richtung der Schichtnormalen, $I_{x}=$ Schichtstrom und $f=$ Streufaktor, hier zu $3 \pi / 8$ angenommen).

Nauk SSSR 110, 970 [1956] (engl. Übers. Soviet Phys. Dokl. 1, 604 [1956]).

5 F. Eckart u. G. Jungk, Ann. Phys., Lpz. 7, 210 [1961].

6 S. Tolansky, Multiple Beam Interferometry, Oxford University Press, London 1948. 